\title{
Patología psiquiátrica asociada al alcoholismo
}

\author{
Casas, M.*; Guardia, J.** \\ * Servicio de Psiquiatría del Hospital Valle Hebrón. Barcelona. \\ * Unidad de Conductas Adictivas del Hospital de Sant Pau. Barcelona.
}

Enviar correspondencia a: Dr. José Guardia. Unidad de Conductas Adictivas. Hospital de la Santa Creu i Sant Pau. C/. San Antonio M M Claret 167. 08025 Barcelona (Spain). Tels. 932919 131-932 919 180. Fax 932919 178. E-mail: jguardia@hsp.santpau.es

\section{RESUMEN}

Los pacientes alcohólicos suelen presentar otros síndromes psiquiátricos asociados, sobretodo de ansiedad y depresión, que con frecuencia son trastornos inducidos o agravados por el propio consumo de alcohol y que tienden a mejorar en pocas semanas, cuando el paciente ha efectuado un tratamiento de desintoxicación y consigue mantenerse en remisión de su alcoholismo.

Sin embargo, en ocasiones se trata de trastornos psiquiátricos independientes, que además del tratamiento del alcoholismo requieren un tratamiento específico. Se trata de la llamada patología dual, en la que concurren uno o varios trastornos psiquiátricos, asociados a la patología adictiva y en la que el alcoholismo suele estar asociado al abuso o dependencia de otras sustancias (cocaína, opiáceos, benzodiazepinas, etc.).

El paciente alcohólico que presenta una patología psiquiátrica severa tiene un mayor riesgo de problemas psico-sociales, recaída y suicidio. Su tratamiento puede ser decisivo para evitar la agravación progresiva y un posible fatal desenlace.

Dichos pacientes requieren un abordaje integrado de su patología dual, en una unidad especializada, con un seguimiento intensivo y durante un tiempo más prolongado, de lo habitual. El programa de intervención debería trabajar con la motivación del paciente para conseguir tanto su buena disposición hacia el abandono del consumo de sustancias, como la estabilización de su patología psiquiátrica (con la ayuda de farmacoterapia) y también el aprendizaje de estrategias de afrontamiento, orientadas hacia la prevención de recaídas.

\section{SUMMARY}

Alcoholic patients have psychiatric syndromes associated, mostly anxiety and depression. Psychiatric syndromes are usually induced by heavy drinking, and tend to improve in a few weeks, when the patient has been detoxified, and a stable remission of alcoholism has been achieved.

However, sometimes alcoholic patients suffer independent psychiatric disorders, that warrant an specific treatment, in addition to the treatment of alcoholism. They are considered dual disorders, with one or more psychiatric disorders, associated to addictive pathology, including abuse or dependency of some other substances (cocaine, opiates, benzodiazepines), in addition to alcohol.

Alcoholic patients with other severe psychiatric disorders may be at greater risk of psychosocial problems, relapse, and suicide. Their treatment can be important in order to stop progressive worsening, associated morbidity and increased mortality risks.

These patients need an integrated treatment of their dual disorder, in specialized units, with intensive follow-up, and for longer periods of time than usual. Treatment programs should deal with patient's motivation, in order to enhance their decision to leave substance use, to stabilize their psychiatric disorder (with pharmacotherapy) and to train in relapse prevention coping skills.

Key words: alcoholism, dual disorders, relapse, suicide, integrated treatment.

Palabras clave: alcoholismo, patología dual, recaída, suicidio, tratamiento integrado. 


\section{INTRODUCCION}

$\mathbf{L}$ a presencia de dos o más trastornos mentales en el mismo paciente recibe el nombre de comorbilidad. Cuando dicha comorbilidad es debida a la concurrencia de un trastorno por abuso de sustancias, asociado a otro trastorno psiquiátrico, recibe la denominación de trastorno dual o diagnóstico dual.

Los pacientes con diagnóstico dual están más discapacitados y requieren más recursos terapéuticos que los que sólo tienen un diagnóstico por abuso de sustancias $u$ otro tipo de trastorno psiquiátrico aislado. Además, tienen un mayor riesgo de suicidio, de quedarse sin hogar, de tener otros problemas legales o médicos y de hospitalizaciones más prolongadas y frecuentes (1).

Las dificultades diaagnósticas que plantean estos pacientes repercuten en que el tratamiento no llegue a ser completo. Además, los centros de tratamiento suelen estar especializados en psiquiatría o bien en drogodependencias, pero no en ambas disciplinas a la vez, con lo cual el paciente difícilmente obtiene la respuesta terapéutica apropiada e incluso puede quedar fuera del sistema asistencial.

\section{EPIDEMIOLOGIA DE LOS TRASTORNOS PSIQUIÁTRICOS}

Los pacientes psiquiátricos tienen un riesgo aumentado de desarrollar drogodependencias y los pacientes drogodependientes también tienen un mayor riesgo de presentar otros trastornos psiquiátricos. Aproximadamente una tercera parte de los pacientes psiquiátricos presentan también abuso de alguna sustancia, en algún momento de su vida, el doble de la proporción esperada para la población general. Por otro lado, más de la mitad de los pacientes drogodependientes han presentado algún otro trastorno psiquiátrico a lo largo de su vida (2).

A partir del estudio E.C.A. ("Epidemiological Catchment Area") se estudiaron 20.000 personas residentes en 5 grandes ciudades de EEUU de América, entre 1980 y 1985.

El estudio ECA encontró un prevalencia de vida del 13 '5\% para el alcoholismo (trastorno por abuso o dependencia del alcohol), del $6^{\prime} 1 \%$ para el abuso o dependencia de otras drogas y un 22 ' $5 \%$ para los demás trastornos psiquiátricos (2) (Figura 1). Asociando los trastornos psiquiátricos y todos los trastornos

\section{Figura 1. Trastornos psiquiátricos en la población general de EEUU (prevalencia de vida)}

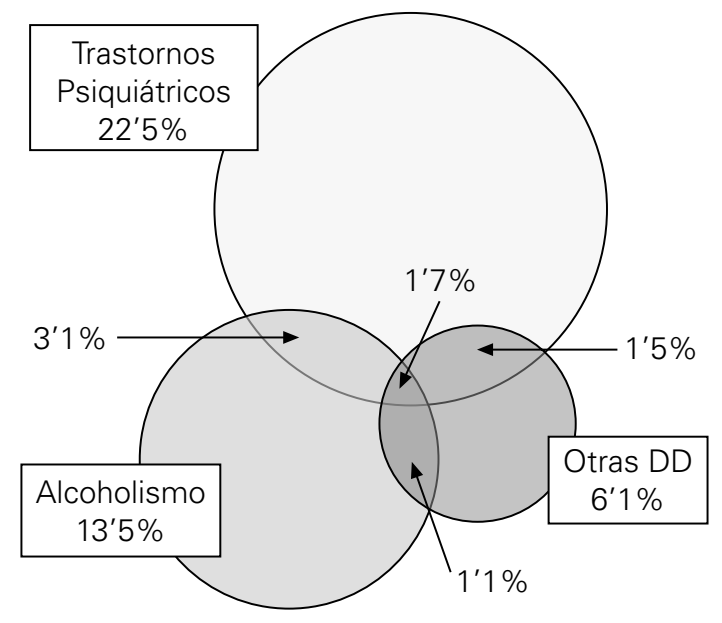


por abuso de sustancias, la prevalencia de vida de trastornos mentales llegaría al 33\% de la población de EEUU (Tablas 1 y 2).

Entre los abusadores de sustancias, la prevalencia de vida de trastornos psiquiátricos sería todavía más elevada que en la población general. Un 36'6\% de las personas con alcoholismo y un $53^{\prime} 1 \%$ de las personas con otras drogodependencias presentaron además algún trastorno psiquiátrico a lo largo de su vida, porcentajes superiores al $22{ }^{\prime} 5 \%$ de la población general (Tabla 3). Y también se detectan una mayor prevalencia de alcoholismo y de otras drogodependencias, entre los pacientes esquizofrénicos, antisociales, ansiosos y afectivos, comparados a la población general (Tabla 4) (2).

Tabla 1. Prevalencia de vida de trastonos mentales en la población general de EEUU (Helzer y Pryzbeck, 1988)

\begin{tabular}{|lcc|}
\hline & \multicolumn{2}{c|}{ Prevalencia de vida (en \%) } \\
\hline & Por diagnósticos & Agrupados \\
\hline Esquizofrenia & $1^{\prime} 5$ & \\
Trastornos afectivos & $8^{\prime} 3$ & \\
Trastornos por ansiedad & $14^{\prime} 6$ & \\
Trastorno Personalidad Antisocial & $2^{\prime} 6$ & $22^{\prime} 5$ \\
Deterioro Cognitivo severo & $1^{\prime} 7$ & \\
\hline Trastornos psiquiátricos & & \\
(excepto Drogodependencias) & & $16^{\prime} 7$ \\
\hline Alcoholismo & $13^{\prime} 5$ & $32^{\prime} 7$ \\
Otras Drogodependencias & $6^{\prime} 1$ & \\
\hline Todas las drogodependencias & & \\
\hline Todos los trastornos psiquiátricos & & \\
\hline
\end{tabular}

Tabla 2. Prevalencia de vida de trastornos psiquiátricos en la población general de EEUU (Regier y cols., 1990)

\begin{tabular}{|lccc|}
\hline & $\begin{array}{c}\text { Prevalencia } \\
\text { último mes } \\
\%\end{array}$ & $\begin{array}{c}\text { Prevalencia } \\
\text { últimos } \mathbf{6} \\
\text { meses \% }\end{array}$ & $\begin{array}{c}\text { Prevalencia } \\
\text { de vida } \\
\%\end{array}$ \\
\hline Trastornos psiquiátricos y drogodependencias & $15^{\prime} 7$ & $19^{\prime} 5$ & $32^{\prime} 7$ \\
Trastornos psiquiátricos excepto drogodependencias & 13 & $15^{\prime} 5$ & $22^{\prime} 5$ \\
Todas las Drogodependencias & $3^{\prime} 8$ & $6^{\prime} 1$ & $16^{\prime} 7$ \\
\hline Alcoholismo & $2^{\prime} 8$ & $4^{\prime} 8$ & $13^{\prime} 5$ \\
Otras Drogodependencias & $1^{\prime} 3$ & 2 & $6^{\prime} 1$ \\
\hline Esquizofrenia & $0^{\prime} 7$ & $0^{\prime} 9$ & $1^{\prime} 5$ \\
Trastornos afectivos & $5^{\prime} 2$ & $5^{\prime} 8$ & $8^{\prime} 3$ \\
Trastornos por ansiedad & $7^{\prime} 3$ & $8^{\prime} 9$ & $14^{\prime} 6$ \\
Trastorno Personalidad Antisocial & $0^{\prime} 5$ & $0^{\prime} 8$ & $2^{\prime} 6$ \\
Deterioro Cognitivo severo & $1^{\prime} 7$ & $1^{\prime} 7$ & $1^{\prime} 7$ \\
\hline
\end{tabular}


Tabla 3. Trastornos psiquiátricos en pacientes drogodependientes (Regier y cols., 1990)

\begin{tabular}{|lccccc|}
\hline & \multicolumn{2}{c}{ Alcoholismo } & \multicolumn{2}{c|}{$\begin{array}{c}\text { Otras } \\
\text { Drogodependencias }\end{array}$} & $\begin{array}{c}\text { Población } \\
\text { General }\end{array}$ \\
\hline & $\%$ & O.R. & $\%$ & O.R. & $\%$ \\
\hline Esquizofrenia & $3^{\prime} 8$ & 3.3 & $6^{\prime} 8$ & 6.2 & $1^{\prime} 5$ \\
Trastornos afectivos & $13^{\prime} 4$ & 1.9 & $26^{\prime} 4$ & 4.7 & $8^{\prime} 3$ \\
Trastornos por ansiedad & $19^{\prime} 4$ & 1.5 & $28^{\prime} 3$ & 2.5 & $14^{\prime} 6$ \\
Trastorno Personalidad Antisocial & $14^{\prime} 3$ & 21.0 & $17^{\prime} 8$ & 13.4 & $2^{\prime} 6$ \\
\hline Trastornos Psiquiátricos & $36^{\prime} 6$ & 2.3 & $53^{\prime} 1$ & 4.5 & $22^{\prime} 5$ \\
(Excepto Drogodependencias) & & & & & \\
\hline
\end{tabular}

\begin{tabular}{|c|c|c|c|c|c|c|c|c|c|}
\hline & \multicolumn{2}{|c|}{ Esquizofrenia } & \multicolumn{2}{|c|}{$\begin{array}{l}\text { Personalidad } \\
\text { Antisocial }\end{array}$} & \multicolumn{2}{|c|}{$\begin{array}{l}\text { Trastornos } \\
\text { por Ansiedad }\end{array}$} & \multicolumn{2}{|c|}{$\begin{array}{l}\text { Trastornos } \\
\text { Afectivos }\end{array}$} & \multirow{2}{*}{$\begin{array}{c}\begin{array}{c}\text { Población } \\
\text { General }\end{array} \\
\%\end{array}$} \\
\hline & $\%$ & O.R. & $\%$ & O.R. & $\%$ & O.R. & $\%$ & O.R. & \\
\hline Alcoholismo & $33^{\prime} 7$ & 3.3 & $73^{\prime} 6$ & 21.0 & $17^{\prime} 9$ & 1.5 & $21^{\prime} 8$ & 1.9 & $13^{\prime} 5$ \\
\hline Otras drogodependencias & $27^{\prime} 5$ & 6.2 & $42^{\prime} 0$ & 13.4 & $11^{\prime} 9$ & 2.5 & $19^{\prime} 4$ & 4.7 & $66^{\prime} 1$ \\
\hline Todas las Drogodependencias & $47^{\prime} 0$ & 4.6 & $83^{\prime} 6$ & 29.6 & $23^{\prime} 7$ & 1.7 & $32^{\prime} 0$ & 2.6 & $16^{\prime} 7$ \\
\hline
\end{tabular}

Un estudio más reciente, el N.C.S. ("National Comorbidity Survey") ha utilizado entrevistas psiquiátricas estructuradas en una muestra de más de 8000 personas no institucionalizadas, de 15 a 54 años de edad y ha encontrado una mayor prevalencia de vida para cualquier trastorno psiquiátrico, un $48 \%$ de la población de EEUU. También se ha detectado una prevalencia más elevada de trastornos psiquiátricos entre las personas que tienen un trastorno por abuso de sustancias que entre los que nunca lo han tenido. Además, proponen que el inicio de la mayoría de dichos trastornos psiquiátricos habría sido previo al inicio del abuso de sustancias, excepto para los trastornos afectivos, como depresión, entre los hombres alcohólicos, cuyo inicio suele ser posterior al inicio del alcoholismo (3).

Entre las mujeres, el $72^{\prime} 4 \%$ de las que abusan del alcohol y el $86 \%$ de las dependientes del alcohol han presentado algún trastorno psiquiátrico o drogodependencia, a lo largo de su vida. Entre los hombres, la prevalencia de vida era de $56{ }^{\prime} 8 \%$ para el abuso y 78 3\% para la dependencia del alcohol.

Los trastornos de ansiedad y de ánimo fueron los más frecuentes entre las mujeres, mientras que las otras drogodependencias y el trastorno de personalidad antisocial fueron los trastornos comórbidos más frecuentes entre los hombres alcohólicos. Para ambos sexos la dependencia del alcohol aparece asociada a trastornos de ansiedad, de estado de ánimo y trastorno de personalidad antisocial, con mayor frecuencia de lo que cabría esperar por simple azar (3).

\subsection{Trastornos psiquiátricos asociados al alcoholismo}

Según el estudio ECA, los trastornos psiquiátricos que aparecen asociados con mayor frecuencia al alcoholismo, son trastornos de 
Tabla 5. Diagnósticos psiquiátricos en pacientes alcohólicos (Helzer y Pryzbeck, 1988).

\begin{tabular}{|lcc|}
\hline & $\begin{array}{c}\text { Prevalencia de vida } \\
\text { Población General }\end{array}$ & $\begin{array}{c}\text { Comorbilidad } \\
\text { Alcoholismo }\end{array}$ \\
\cline { 2 - 3 } & $\%$ & O.R. \\
\hline Personalidad antisocial & $2^{\prime} 6$ & 21.0 \\
Otras Drogodependencias & $6^{\prime} 1$ & 7.2 \\
Mania & $0^{\prime} 4$ & 6.2 \\
Esquizofrenia & $1^{\prime} 5$ & 4.0 \\
Trastornos Pánico & $1^{\prime} 5$ & 2.4 \\
Trastorno obsesivo compulsivo & $2^{\prime} 5$ & 2.1 \\
Distimia & $1^{\prime} 5$ & 1.8 \\
Depresión Mayor & $5^{\prime} 1$ & 1.7 \\
Trastorno por Somatización & $0^{\prime} 1$ & 1.8 \\
Trastornos fóbicos & $12^{\prime} 6$ & 1.4 \\
Anorexia & $0^{\prime} 1$ & 1.2 \\
Deterioro Cognitivo & $1^{\prime} 7$ & 0.4 \\
\hline
\end{tabular}

personalidad antisocial (21 veces más probable que en la población general), la manía (6'2 veces más), la esquizofrenia (4 veces más) y el abuso de drogas (3'9 veces más) (Tabla 5). Y las drogodependencias que aparecen asociadas al alcoholismo, por orden de mayor a menor frecuencia serían las de cocaína, hipnosedativos, opiáceos, alucinógenos, estimulantes y cannabis (Tabla 6).

Aunque los síntomas aislados de ansiedad y depresión aparecen asociados con frecuencia al alcoholismo, los trastornos depresivos o de ansiedad no son mucho más prevalentes que entre la población general (4).

En la población general, los trastornos de ansiedad son los trastornos psiquiátricos más prevalentes, llegando, según algunos estudios hasta el $25 \%$ de la población (3). Entre un $23 \%$ y un $70 \%$ de pacientes alcohólicos presentan también trastornos de ansiedad, sobretodo neurosis de ansiedad y fobias. Por otro lado, del $20 \%$ al $45 \%$ de pacientes con trastorno de ansiedad tiene antecedentes de alcoholismo (5). Dicha comorbilidad entre alcoholismo y trastornos de ansiedad es más prevalente entre los dependientes que entre los abusadores de alcohol.

\begin{tabular}{|lc|}
\hline $\begin{array}{c}\text { Tabla 6. Otras drogodependencias } \\
\text { en pacientes alcohólicos } \\
\text { (Helzer y Pryzbeck, 1988) }\end{array}$ \\
\hline & $\begin{array}{c}\text { Comorbilidad } \\
\text { Alcoholismo }\end{array}$ \\
\hline & $\mathbf{O . R .}$ \\
\hline Cocaína & 35.0 \\
Sedativos & 17.0 \\
Opiáceos & 13.0 \\
Alucinógenos & 12.0 \\
Estimulantes & 11.0 \\
Cannabis & 6.0 \\
\hline
\end{tabular}

Entre los trastornos psiquiátricos de Eje II, asociados al alcoholismo, destacan los trastornos de personalidad (57-78\%), que se distribuyen entre los de tipo paranoide (7-44\%), antisocial (3-47\%), límite (16-32\%), histriónico $(6-34 \%)$, por evitación (2-32\%), y dependiente (4-29\%). En función de la tipología de alcoholismo, la comorbilidad de Eje I es dos veces más frecuente en el tipo A que en el tipo B. Sin embargo, la comorbilidad con trastronos de personalidad (acompañada o no por 
algún trastorno de Eje I), es más frecuente en los pacientes alcohólicos de tipo $B$ que en los de tipo A. Sobretodo los trastorno de personalidad esquizoide, esquizotípico, o los del cluster B (6).

\section{ETIOPATOGENIA DE LOS TRASTORNOS PSIQUIÁTRICOS ASOCIADOS AL ALCO- HOLISMO}

Los trastornos psiquiátricos podrían conducir al alcoholismo. Los pacientes con síntomas psiquiátricos podrían recurrir a beber, como auto-medicación de sus síntomas de ansiedad. En tal caso, el trastorno psiquiátrico precedería al alcoholismo en varios años y el alcoholismo sería secundario (7).

Pero, por otro lado, el consumo excesivo de alcohol contribuye al desarrollo de síntomas psiquiátricos, como el deterioro de la expresión emocional, síntomas de ansiedad, depresión, y trastornos de conducta que producen desadaptación social. Se trata de síntomas o síndromes psiquiátricos, inducidos por el consumo excesivo de alcohol o por su abstinencia, en el contexto de un alcoholismo primario. Estos síntomas o síndromes psiquiátricos inducidos por el alcohol tenderán a su remisión espontánea, pocos días o semanas después de haber abandonado el consumo de alcohol.

Los pacientes alcohólicos refieren con frecuencia que beben para mitigar o aliviar estados de ánimo disfórico, lo cual ha sido considerado como "auto-medicación." Sin embargo, el consumo crónico de alcohol y su posible abstinencia pueden agravar estados de ámino negativo, como consecuencia de los efectos farmacológicos del alcohol, o bien de los problemas psicosociales asociados.

Los pacientes que presentan un trastorno bipolar pueden beber para aliviar tanto los síntomas depresivos como maníacos, pero la evidencia indica que el mayor riesgo para el consumo excesivo se produce durante la fase maníaca de su enfermedad. Los pacientes con agorafobia o fobia social pueden beber para aliviar sus síntomas de ansiedad, sin embargo, los que padecen un trastorno de angustia o de ansiedad generalizada pueden experimentar dichos síntomas como consecuencia del consumo excesivo de alcohol (8).

Por tanto, determinados trastornos psiquiátricos pueden ser inducidos por la intoxicación o la abstinencia de sustancias psicotrópicas. De hecho, la clasificación diagnóstica psiquiátrica DSM-IV considera como trastornos psiquiátricos inducidos por el alcohol (9).

-Delirium (por intoxicación y por abstinencia)

-Demencia persistente

-Trastorno Amnéstico persistente

-Trastorno psicótico (con ideas delir. o con alucin.)

-Trastorno del estado de ánimo

- Trastorno de ansiedad

-Trastorno sexual

-Trastorno del sueño

\subsection{Estudios de antecedentes familiares}

Si dos trastornos tienen una relación causal, es decir, cuando uno de ellos es consecuencia del otro, los familiares de los probandos presentarán un riesgo aumentado para el trastorno causal y también para la combinación de ambos trastornos, pero no para la forma pura del trastorno secundario. Por ejemplo, si el alcoholismo es secundario a la ansiedad, los familiares de probandos con ansiedad deberían presentar una mayor tasa de ansiedad aislada o bien asociada al alcoholismo, pero una tasa normal de alcoholismo.

Sin embargo, si ambos trastornos comparten una etiología común (neurobiológica o ambiental), es decir que ambos trastornos son consecuencia de un tercer factor; los familiares de los probandos con uno de los dos trastornos presentarán también elevadas tasas de la forma pura del otro trastorno, en comparación a la población general. Es decir que si la ansiedad y el alcoholismo comparten una etiología común, los familiares de probandos con ansiedad deberían presentar 
un riesgo aumentado de alcoholismo y viceversa.

Los estudios de familiares deben incluir un número suficiente de probandos, con formas puras de cada trastorno, así como sujetos control que no presenten ninguno de los trastornos.

Mediante este procedimiento se han detectado factores de susceptibilidad compartida para el alcoholismo y el trastorno de pánico, ya que los familiares de probandos con trastorno de pánico aislado también presentan un elevado riesgo de alcoholismo. Pero la conclusión del estudio es que el alcoholismo y la ansiedad pueden ser transmitidos de manera independiente, dentro de los familiares y que cuando los trastornos de ansiedad y el alcoholismo son comórbidos pueden ser también cotransmitidos (7).

\subsubsection{Estudio de antecedentes familiares sobre la comorbilidad entre alcoholis- mo y ansiedad}

El estudio de Yale incluyó 226 probandos, distribuídos en cuatro grupos, los que presentaban un trastorno por dependencia del alcohol, los que presentaban un trastorno de ansiedad, los que presentaban ambos trastornos (dependencia del alcohol y ansiedad) y los que no presentaban ningún trastorno del eje I, ni tampoco un trastorno de personalidad antisocial.

Los familiares de los probandos con un trastorno de ansiedad presentaron un riesgo aumentado para el desarrollo de ambos trastornos y más aumentado todavía cuando el probando presenta la comorbilidad alcoholismo y ansiedad asociados.

La presencia de un trastorno de ansiedad en el probando parece aumentar el riesgo para la dependencia del alcohol en los familiares, pero la dependencia del alcohol en los probandos no aumentó el riesgo de los familiares para los trastornos de ansiedad, lo cual sugiere que algunos factores etilógicos pueden ser compartidos y que incluyen factores genéticos, ambientales o de exposición pre- natal, como el consumo materno de alcohol, que puede predisponer para ambos trastornos.

A partir de un estudio con mujeres gemelas, se ha comprobado que la etiología de los trastornos de ansiedad y el alcoholismo, en las mujeres, podría ser parcialmente atribuíble a factores genéticos comunes de vulnerabilidad compartida para ambos trastornos (10).

Los parientes masculinos dependientes del alcohol tenían el doble de posibilidades de presentar un trastorno de ansiedad que los parientes varones no dependientes del alcohol; mientras que en las parientes mujeres alcohol-dependientes la probabilidad era 3'7 veces mayor.

Las probabilidades de presentar un trastorno psiquiátrico comórbido pueden variar en función del sexo. Por ejemplo, para el trastorno de pánico, la "odds ratio" (o razón de ventaja) es del 0'6 para los varones alcohólicos, pero llega al 4'2 en el caso de las mujeres. Y con respecto al trastorno de ansiedad, la probabilidad de que un pariente dependiente del alcohol presente un trastorno de ansiedad asociado, suele ser también mayor en las mujeres (3'7 veces mayor) que en los varones (2 veces mayor) (7).

\subsection{Comorbilidad y cotransmisión de la de- pendencia frente al abuso de alcohol}

La dependencia del alcohol, en los probandos se asocia con más frecuencia a la dependencia que al abuso de alcohol, en los familiares (7). La probabilidad de heredar un trastorno por dependencia del alcohol sería mayor que la de heredar un trastorno por abuso de alcohol, por lo que se podría considerar que el abuso podría estar más relacionado con factores ambientales, de tal manera que la dependencia del alcohol tendería a agruparse en determinados familiares, mientras que el abuso no tiene por qué hacerlo.

Los trastornos de ansiedad en los probandos también se asocian a la dependencia del 
alcohol, en los familiares, pero no al abuso de alcohol. Por tanto, la dependencia del alcohol y los trastornos de ansiedad pueden ser consecuencia de factores de riesgo subyacentes compartidos, que podrían ser en parte genéticos (10).

El alcoholismo puede estar asociado a trastornos afectivos y trastornos de ansiedad. La comorbilidad es más frecuente en mujeres. Los pacientes alcohólicos con ansiedad comórbida experimentan una abstinencia del alcohol más severa y una mayor tendencia a la recaída. Por otro lado, la abstinencia del alcohol puede mimetizar los síntomas de angustia y de ansiedad generalizada.

Los niños que experimentan niveles elevados de ansiedad tienen un riesgo más elevado de utilizar el alcohol como auto-medicación de dichos síntomas de ansiedad, en la vida adulta. Por tanto, su identificación y tratamiento precoces podría ayudar a prevenir un potencial abuso o dependencia del alcohol, en el futuro (7).

\subsection{Relación de causalidad entre el tras- torno psiquiátrico y elalcoholismo}

La posible relación de causalidad entre el alcoholismo y los trastornos psiquiátricos asociados se puede producir de maneras diversas:

1. El alcoholismo y el trastorno psiquiátrico pueden ser simultáneos o sucesivos y su asociación se puede dar por coincidencia.

2. El alcoholismo puede ser la causa o bien aumentar la gravedad de determinados trastornos psiquiátricos

3. Los trastornos psiquiátricos pueden ser la causa o bien aumentar la gravedad del alcoholismo.

4. Tanto el alcoholismo como los trastornos psiquiátricos pueden ser la consecuencia de una tercera condición.

5. Tanto el consumo excesivo como la abstinencia de alcohol pueden inducir síntomas muy parecidos a los de un trastorno psiquiátrico independiente, que son los que el DSM-IV considera como trastornos inducidos por intoxicación o por abstinencia del alcohol (11).

Los estudios clínicos con pacientes alcohólicos sugieren que la mayoría de trastornos psiquiátricos comórbidos son secundarios al alcoholismo y remiten espontáneamente con el tratamiento de desintoxicación del alcohol y sin necesidad de un tratamiento psiquiátrico adicional (1).

El consumo excesivo de alcohol puede inducir nuevos síntomas psiquiátricos o exacerbar síntomas psiquiátricos independientes. Sobretodo con referencia a los síntomas de ansiedad y depresión, inducidos por el consumo excesivo o la abstinencia del alcohol. Sin embargo, dichos síntomas remiten espontáneamente con la abstención continuada, lo cual sugiere que no se trata de trastornos psiquiátricos independientes (1).

La hipótesis de la auto-medicación sugiere que algunas personas consumirían alcohol para aliviar síntomas de ansiedad, tensión, depresión, insomnio, apatía y aislamiento social; asociados a trastornos mentales. De esta manera, personas con trastornos mentales podrían persistir en el consumo de alcohol, a pesar de que empeore sus síntomas, tal vez por déficit de introspección o de aprendizaje por la experiencia Este consumo por auto-medicación podría desembocar en un verdadero alcoholismo.

Sin embargo, el metanálisis efectuado por Berglund y Öjenhagen (12) de un total de 42 artículos publicados entre 1994 y 1996, concluye que los datos obtenidos dan poco apoyo a la validez de la hipótesis sobre automedicación y que el supuesto alivio de los síntomas psiquiátricos, tras el consumo de alcohol, podría ser mejor explicado por sus expectativas de efectos positivos del alcohol, más que por un verdadero efecto terapéutico del consumo de alcohol. Además, los pacientes con enfermedades psiquiátricas que abusan del alcohol tienden a presentar una peor evolución de su trastorno psiquiátrico, mientras que un consumo leve o moderado no 
tiene efectos positivos documentados sobre los trastornos psiquiátricos.

\subsection{Estrés, alcoholismo y otros trastornos psiquiátricos}

La exposición a situaciones estresantes es una experiencia humana que se repite con frecuencia. El estrés intenso puede inducir alteraciones fisiológicas y conductuales que van desde los trastornos psiquiátricos hasta la disfunción del sistema inmunológico.

Determinadas experiencias estresantes pueden inducir síntomas depresivos, mientras que otras pueden generar ansiedad. Algunas son breves y transitorias pero otras pueden ser persistentes o tener efectos retardados o desencadenar otras pérdidas (económicas o de apoyo social) que van a empeorar la situación de la persona.

El estrés incontrolable puede llegar a inducir un estado de "indefensión aprendida", con modificaciones en la neurotransmisión que pueden aumentar la vulnerabilidad individual hacia la psicopatología. La capacidad para afrontar y resolver los problemas puede tener un efecto amortiguador del impacto del estrés.

Las experiencias vitales tempranas de exposición al estrés pueden inducir sensibilización y respuesta aumentada ante las situaciones estresantes, lo cual podría estar en relación con las elevadas tasas de recaída de algunos trastornos psiquiátricos como la depresión. Los psicoestimulantes, el alcohol y otras sustancias pueden inducir también efectos de sensibilización y respuestas alteradas del eje hipotálamo-hipofisario-suprarrenal.

La mayor vulnerabilidad hacia el alcoholismo puede estar en relación con una predisposición genética, pero también las experiencias repetidas de estrés intenso pueden aumentar el riesgo de padecer tanto una drogodependencia como otros trastornos psiquiátricos.

La genética y el estrés actuarían facilitando la progresión del proceso de neuroadaptación al alcohol, que sería más intenso y permanente; aumentando de esta manera el riesgo de desarrollar alcoholismo, bien sea por mecanismos hereditarios o bien adquiridos.

Dichas experiencias de estrés pueden ser mediadas tanto por factores internos (como un trastorno psiquiátrico) como por agresiones ambientales (como un traumatismo psíquico o la pérdida de un miembro de la familia).

Además, uno de los neurotransmisores implicados en los efectos del estrés sobre el proceso de neuroadaptación y sensibilización es la serotonina, que también se encuentra implicada con los trastornos afectivos y los de ansiedad.

Por otro lado, los pacientes alcohólicos en recuperación precoz suelen presentar una baja tolerancia al estrés, y las situaciones estresantes van a aumentar el riesgo de recaída, activando el circuíto que implica a la amígdala, el córtex frontal dorso-lateral, y los ganglios basales, lo cual se puede manifestar en forma de respuestas de craving, ante dichas situaciones estresantes (13).

\section{CARACTERÍSTICAS CLÍNICAS}

Según el tipo de comorbilidad asociada al alcoholismo, las características clínicas del trastorno global pueden ser diferentes.

\subsection{Alcoholismo y depresión}

El consumo excesivo continuado de bebidas alcohólicas podría inducir estados depresivos graves pero transitorios, en cualquier persona que no tenga antecedentes de depresión $(14,15)$. Los síntomas depresivos remiten rápidamente durante las 4-6 semanas posteriores a la desintoxicación (16). A las 3 semanas de abstinencia, el grupo de pacientes alcohólicos primarios que presentaba depresión secundaria mostraba una reducción del 49\% de los síntomas depresivos; mientras que el grupo de pacientes que presentaba depresión primaria y alcoholismo secundario sólo tuvo una reducción del 14\%. 
Ningún estudio ha demostrado que los trastornos depresivos pueden ser la causa del alcoholismo. Sin embargo, el consumo excesivo continuado de alcohol puede inducir síntomas afectivos transitorios, incluso en personas que no tenían antecedentes de depresión (17). Por otro lado, la depresión puede formar parte del proceso de recuperación de cualquier conducta adictiva. El hecho de tener que renunciar al consumo de alcohol o la pérdida de relaciones interpersonales significativas puede resultar emocionalmente doloroso.

El 80\% de pacientes alcohólicos presentan algún episodio depresivo mayor, a lo largo de su vida $(11,16)$, que suele ser la consecuencia del consumo excesivo, más que la presencia de un trastorno depresivo independiente, sobretodo en varones alcohólicos primarios (14).

Para efectuar el diagnóstico diferencial entre el trastorno depresivo primario y el inducido es importante explorar la presencia de antecedentes de un posible trastorno afectivo, previo al inicio del alcoholismo, ansiedad de separación en la infancia, fobias o trastornos de ansiedad, reacción hipomaníaca a los antidepresivos, o antecedentes familiares de trastorno bipolar.

La depresión, asociada al alcoholismo predice pobres resultados para el tratamiento del alcoholismo, por lo menos en varones (18) y un aumento del riesgo de conductas suicidas (19). Los estados emocionales negativos son el factor de recaída más habitual. Los episodios depresivos pueden aumentar el riesgo de recaída, mientras que la remisión de la depresión predice un menor riesgo de recaída en el consumo de alcohol (20)

Cuando un trastorno psiquiátrico (como la depresión), ha sido inducido por el consumo excesivo de alcohol, también va a mejorar con la abstinencia continuada de bebidas alcohólicas (21). El consumo excesivo de alcohol interfiere con la recuperación de otro trastorno psiquiátrico asociado, (como depresión) y puede generar lo que se ha dado en llamar un trastorno psiquiátrico refractario al tratamiento, incluso cuando dicho trastorno recibe el tratamiento farmacológico apropiado (como antidepresivos).

En el estudio TROMSO, un estudio epidemiológico sobre enfermedad cardiovascular, efectuado en Noruega, el consumo excesivo de alcohol predice un estado de ánimo depresivo (en ambos sexos). El ánimo depresivo predice aumento del consumo en bebedores excesivos (en ambos sexos y en varones bebedores moderados). Pero, en las mujeres bebedoras moderadas, predice una reducción del consumo.

\subsection{Alcoholismo y suicidio}

Cuando aumenta la venta y el consumo de alcohol, en un determinado país, tienden a aumentar también las tasas de suicidio y de homicidio. Parecen ser más bien las bebidas destiladas las que estarían asociadas al suicidio, mientras que el consumo de bebidas fermentadas no parece influir en la tasa de suicidios.

Los dos trastornos mentales que con mayor frecuencia se asocian al suicidio son la enfermedad depresiva y el alcoholismo. El alcoholismo es más frecuente entre los suicidios de varones $y$, particularmente, entre los alcohólicos de inicio precoz.

La muerte por suicidio es más frecuente en los pacientes alcohólicos (5\%-27\%) que en la población general (1\%). Un 15\%-25\% de todos los suicidios se producen en pacientes alcohólicos. Las pérdidas sociales recientes, los efectos depresógenos, tóxicos y desinhibidores del alcohol, los síntomas persistentes de depresión y los rasgos de personalidad, pueden contribuir a los gestos suicidas. Otros factores que aumentan el riesgo suicida son la edad avanzada, estado separado o viudo, desempleo o jubilación, enfermedad somática y polidrogodependencia (12).

Un 18-20\% de alcohólicos han intentado el suicidio, en alguna ocasión (19). El suicidio consumado es más frecuente en varones (23 por cada mujer). Las mujeres (37\%) tienen 
más intentos de autolisis que los hombres $(13 \%)(22)$.

La probabilidad de suicidio entre los alcohólicos es 60-120 veces mayor que en la población general. Entre las personas que se suicidan, el 15-30\% son pacientes alcohólicos. La depresión y el alcoholismo, se encuentran entre las primeras causas del suicidio y con frecuencia están asociados. El 90-94\% de suicidas presentan un trastorno psiquiátrico. Entre ellos, el 47-70\% presentan un trastorno afectivo, el $15-25 \%$ presentan alcoholismo y el alcohol precipita el suicidio en el $68 \%$ de los casos.

Al comparar los rasgos de personalidad, entre los alcohólicos que presentan intentos de autolisis, con los que no los han tenido, se aprecia una mayor psicopatología, impulsividad, rasgos paranoides, ansiedad, aceleración psicomotriz y conductas extravagantes; a la vez que una menor seguridad y más baja auto-estima (22).

\subsection{Alcoholismo y trastornos de ansiedad}

Schuckit y colaboradores (17) establecen la diferenciación entre trastornos psiquiátricos concurrentes e independientes. Los pacientes alcohólicos presentan una elevada prevalencia de trastornos afectivos y de ansiedad, pero la mayoría de ellos son concurrentes.

En función de la prevalencia de vida, los trastornos de ansiedad, entre los pacientes alcohólicos se distribuyen en agorafobia con trastorno de pánico (31'5\%), trastorno obsesivo-compulsivo (24'6\%), fobia social (21'9\%), trastorno de pánico $\left(20^{\prime} 4 \%\right)$, fobia simple (14'4\%) y agorafobia (sin trastorno de pánico) (13'3\%) (23).

Ross y colaboradores (24) encuentran la prevalencia más elevada para el trastorno por ansiedad generalizada (50'7\%), seguida por los trastornos fóbicos (30'5\%). Schuckit y colaboradores (17) consideran que únicamente el trastorno de pánico y la fobia social serían más prevalentes, como trastornos de ansiedad independientes y que entre los tras- tornos independientes, sólo habría un riesgo aumentado para el trastorno bipolar, trastorno por angustia y fobia social, entre los alcohólicos primarios, con antecedentes familiares de alcoholismo. Los trastornos psiquiátricos independientes serían los que se inician antes del alcoholismo o que permanecen durante períodos de abstinencia de más de 3 meses y se acompañan de antecedentes del mismo trastorno, en sus familiares.

El alcoholismo puede inducir trastornos de ansiedad. Tal vez en personas vulnerables y en relación con las pérdidas de relaciones, fracaso formativo o vocacional, acontecimientos vitales o episodios repetidos de abstinencia (hiperadrenérgicos) (5). Una disregulación noradrenérgica que es todavía mayor en los que además del alcohol abusan de la cocaína.

Por otro lado, los pacientes con trastorno de ansiedad presentan 2' 5 a 4'3 veces más riesgo para el alcoholismo que la población general. Según un estudio prospectivo reciente, existe una relación causal recíproca entre los trastornos de ansiedad y el alcoholismo. Los trastornos de ansiedad tendrían una mayor probabilidad de desarrollar alcoholismo y viceversa (5).

Stockwell (25) destaca los síntomas afectivos (miedo, ansiedad) dentro del Síndrome de abstinencia del alcohol. El efecto de rebote de ansiedad e insomnio serían poderosos estímulos condicionados, que inducirían a un nuevo consumo de alcohol.

Los síntomas de abstinencia del alcohol pueden confundirse con la ansiedad generalizada o con crisis de angustia. Esto puede llevar a la prescripción de benzodiazepinas, cuyo consumo continuado produce efectos de rebote, empeora la severidad de la abstinencia, la intensidad de la dependencia y el pronóstico de su recuperación, sobretodo cuando son de vida media corta y cuando tienen un efecto reforzador. Hay muchas evidencias de que incluso el consumo moderado de alcohol puede interferir en la recuperación de un trastorno afectivo o de ansiedad. Por tanto, lo prudente es recomendar la abstinencia del alcohol a dichos pacientes, (por lo menos durante su recuperación). 
El curso del alcoholismo influye claramente en el curso de la depresión. La remisión del alcoholismo aumenta claramente la probabilidad de remisión de la depresión e incluso tiene un cierto efecto protector de la recaída, en el trastorno depresivo (20).

\subsection{Alcoholismo y trastornos de personali- dad}

La prevalencia de trastornos de personalidad en pacientes alcohólicos se ha situado entre un 15 y un $25 \%$ y la de alcoholismo en los trastornos de personalidad alcanza el $50 \%$ al $75 \%$ (26).

El alcoholismo induce trastornos de conducta, que pueden acompañarse de violencia verbal o física, no respetar los derechos de los demás, mentiras, falta de honestidad, y otros rasgos de conducta antisocial. Pero, el trastorno de personalidad antisocial se inicia a los 15 años de edad y persiste incluso tras la abstinencia prolongada del alcohol.

El trastorno de personalidad antisocial se asociaría con mayor frecuencia al tipo II de Cloninger o al tipo B de Babor, que se caracterizan por rasgos de conducta antisocial, búsqueda de lo novedoso, baja evitación de lo perjudicial, inicio precoz del abuso de alcohol y de los problemas asociados.

La presencia de conductas antisociales graves, en diversas áreas de funcionamiento, que se inician antes de los 15 años de edad y que persisten durante la vida adulta, es el requisito diagnóstico imprescindible para diferenciarlo de la conducta antisocial secundaria al alcoholismo.

Se trata de personas impulsivas, violentas, que les gusta asumir riesgos e incapaces de aprender de sus errores o de beneficiarse del castigo. Que van a tener dificultades para controlar el consumo de sustancias, ya que la mayoría de ellos van a tener graves problemas con el alcohol secundarios, a lo largo de su vida y también problemas con otras drogas, violencia, interrupción prematura de tratamiento y mal pronóstico. Suelen representar el $5 \%$ de mujeres y el $10-20 \%$ de los hombres que solicitan tratamiento del alcoholismo.

\subsection{Alcoholismo y otras drogodependen- cias}

Cada vez con mayor frecuencia, el alcoholismo aparece asociado al abuso o dependencia de otras drogas. En la población general de Estados Unidos, las personas con dependencia del alcohol tienen 5 veces más probabilidades de tener alguna otra drogodependencia asociada (18\% frente al 3'5\%) (Helzer y Pryzbeck, 1988). Los abusadores de diversas sustancias suelen ser más jóvenes y presentar más problemas relacionados con el alcohol o las drogas (26).

La prevalencia de alcoholismo entre dependientes de cocaína podría ser de hasta el $84 \%$ y entre los dependientes de opiáceos, del $65 \%$ (2).

Los pacientes alcohólicos presentan un mayor riesgo de dependencia de cocaína, hipnosedativos, opiáceos, alucinógenos, estimulantes y cannabis (Tabla 6) (4); siendo la prevalencia de dependencia de nicotina y de benzodiazepinas, claramente superior a la de la población general.

El consumo de otras sustancias puede aumentar el riesgo de recaída en el consumo de alcohol, en los pacientes alcohólicos que se encuentran en recuperación. Por este motivo habrá que ampliar la intervención, al consumo de todo tipo de sustancias, cuando se pretende una completa recuperación del alcoholismo. Además, el síndrome de abstinencia de otras drogas puede iniciarse días después de la abstinencia de alcohol y una persona dependiente de diversas sustancias puede presentar diversas oleadas de abstincia que se van superponiendo (27).

Sin embargo, cuando el paciente acude a solicitar tratamiento, su demanda suele estar centrada en una de las sustancias que toma pero, con frecuencia, no considera que el consumo de otras sustancias le sea perjudicial y no se muestra interesado en cambiar sus patrones de consumo de las otras sus- 
tancias. Conviene aplicar, por tanto, técnicas motivacionales, con la finalidad que el paciente progrese de fase motivacional con respecto a las otras sustancias y llegue a aceptar la necesidad de reducir o abandonar su consumo, que con frecuencia va a requerir un ingreso para la desintoxicación de las diversas sustancias que toma.

Aunque la retirada de todas las sustancias sería el objetivo ideal, se puede hacer una aproximación progresiva, partiendo de objetivos más realistas y que puedan ser aceptados por y pactados con el propio paciente. Por ejemplo, dejar de utilizar la vía intravenosa y pasar a la vía oral, mediante un programa de mantenimiento con metadona. O bien sustituir benzodiazepinas de vida media corta por una pauta decreciente de otro tipo de benzodiazepinas de vida media larga, a la vez que iniciamos una pauta de mantenimiento con anticomiciales (valproato, topiramato, gabapentina, etc.).

Otros objetivos a tener en cuenta serían evitar la prescripción de fármacos potencialmente letales cuando se toma una sobredosis o cuando se toman asociados al consumo excesivo de alcohol. Efectuar determinaciones periódicas de las diversas sustancias en orina, para monitorizar su consumo. Incorporar al paciente a un grupo motivacional o bien de prevención de recaídas. Puede ser útil fijar objetivos intermedios, sencillos de alcanzar, y otros objetivos más ambiciosos, a largo plazo.

También conviene tener en cuenta y transmitir, tanto al paciente como a sus familiares, que el proceso de recuperación va a ser a largo plazo, que se van a producir períodos de remisión (parcial o completa), interrumpidos por episodios de recaída más o menos prolongados, y que la mejoría, en cualquiera de las áreas de funcionamiento del paciente drogodependiente es siempre valiosa, así como también lo es conseguir detener el proceso de agravación progresiva de la dependencia de diversas sustancias que, inevitablemente, induciría otros trastornos médicos y psiquiátricos asociados.

\subsection{Alcoholismo y otras conductas adictivas}

Existen elevadas tasas de comorbilidad entre dependencia del alcohol y ludopatía. Los resultados de un estudio comparativo entre gemelos varones monozigóticos y dizigóticos sugieren que la comorbilidad alcoholismoludopatía se podría atribuir a los factores genéticos más que a los ambientales (28)

Respecto a los trastornos de la conducta alimentaria, según el estudio epidemiológico de comorbilidad de los Estados Unidos existen elevadas tasas de comorbilidad entre las drogodependencias y la bulimia y también entre anorexia restrictiva y abuso de drogas pero no entre anorexia y alcoholismo (29).

La bulimia nerviosa y el subtipo de anorexia que cursa con atracones de comida y comportamiento purgativo, están más frecuentemente asociadas al alcoholismo que la anorexia nerviosa restrictiva. De hecho ambos subgrupos de trastornos de la alimentación podrían tener características de personalidad distintas. Las primeras suelen presentar conducta impulsiva e inestabilidad emocional, mientras que las anoréxicas restrictivas suelen presentar conductas compulsivas y más controladas (30).

Los estudios genéticos de seis trastornos psiquiátricos mayores en mujeres, que incluyen bulimia y alcoholismo, proponen que ambos trastornos estarían en relación con entidades genéticas distintas y serían consecuencia de factores causales independientes (10).

En un estudio japonés con pacientes alcohólicos se detectó el trastorno de la conducta alimentaria en el $11 \%$ de la mujeres y el $0^{\prime} 2 \%$ de los hombres. El inicio del trastorno alimentario se produjo entorno a los 19'7 años de edad y era previo al del alcoholismo, cuyo inicio se situó entorno a los 24'6 años (31).

\subsection{Alcoholismo y otras alteraciones con- ductuales graves}

Una elevada proporción de personas que han cometido delitos con violencia y también 
sus víctimas se encuentran bajo los efectos del alcohol, cuando se produce el incidente violento. Sobretodo cuando se trata de personas jóvenes.

Las personas sin hogar ("homeless"), presentan elevadas tasas de trastornos psiquiátricos y abuso de sustancias. Las mujeres sin techo presentan una elevada prevalencia de esquizofrenia y trastorno bipolar, asociado a drogodependencias. Las madres acogidas en residencias presentan trastorno por estrés postraumático y depresión mayor, asociados a drogodependencias.

Las mujeres sin hogar y también las que viven en contextos sociales de pobreza, tienen mayor riesgo de sufrir violencia y abuso sexual, así como una elevada prevalencia de trastorno de estrés postraumático, depresión mayor y abuso de sustancias (29).

El consumo de alcohol juega un importante papel en la violencia familiar, incluidos los malos tratos a la mujer e hijos, a los ancianos, abuso sexual, violación y homicidio. Los estados de intoxicación alcohólica están también relacionados con accidentes de tráfico, delitos contra la propiedad y otros delitos.

Los pacientes alcohólicos (que habían estado ingresados, al menos 1 vez) tenían 3'5 veces más probabilidades de presentar delincuencia registrada (delitos violentos, contra la propiedad y de tráfico) que los pacientes control (en un estudio suizo). La mayoría presentaban trastornos de personalidad (sobretodo el antisocial), más antecedentes de suicidio y conducta agresiva; más abuso de drogas, inicio más precoz del tratamiento psiquiátrico y eran más jóvenes.

\subsection{Alcoholismo y enfermedad mental grave}

El alcoholismo es el trastorno mental comórbido más frecuente en personas con enfermedad mental grave, como esquizofrenia o trastorno bipolar. La esquizofrenia tiene una mayor prevalencia de vida entre los alcohólicos (3'8\%) que en la población general (1'5\%). Por otro lado, entre los pacientes esquizofrénicos, la prevalencia de alcoholismo $\left(33^{\prime} 7 \%\right)$ es también más elevada que en la población general (13'5\%), según el estudio E.C.A. (2).

Según el estudio ECA, el 33'7\% de esquizofrénicos y el $42^{\prime} 6 \%$ de bipolares reunían criterios de alcoholismo (abuso o dependencia de alcohol), comparado al $16^{\prime} 7 \%$ de la población general (2).

Según el estudio NCS el 9'7\% de personas con manía cumplían criterios de dependencia del alcohol, a lo largo de su vida (3), siendo particularmente frecuentes en jóvenes, pacientes hospitalizados, servicios de urgencias y albergues para personas sin hogar.

Entre los factores etiológicos hay que tener en cuenta que (1) la deriva social que sufren los enfermos mentales graves les lleva a una mayor exposición y más fácil acceso al alcohol y las drogas; (2) puede haber un intento de aliviar o auto-medicar determinados síntomas de la enferemedad mental y los efectos secundarios de la medicación y (3) puede ser también un intento de evitar ser etiquetados como pacientes mentales.

Los enfermos mentales severos con una drogodependencia asociada tienen un elevado riesgo de inestabilidad en su alojamiento e incluso de quedarse sin hogar. Además presentan un mal cumlplimiento de la medicación y son atendidos con frecuencia en los servicios de urgencias, hospitales y prisiones. Además suelen presentar abuso de otras drogas, mal cumplimiento de la medicación, viven en circunstancias estresantes y carecen de redes de apoyo social (32).

Cuando los pacientes mentales graves dejan de beber, presentan muchos signos de mejoría en su bienestar, por tanto se puede deducir que el alcoholismo comórbido empeora la adaptación de los enfermos mentales graves. Su evolución espontánea tiende hacia el empeoramiento, con una elevada proporción de los que se quedan sin hogar y presentan conductas desadaptadas, hospitalizaciones frecuentes y estancias en prisión. Sin embargo, cuando abandonan el consumo de alcohol, mejora su adaptación y su pronósti- 
co. En el estudio ECA, entre los pacientes esquizofrénicos que dejaron de beber, disminuyeron las tasas de depresión y hospitalización, al año de seguimiento (33).

El abuso de alcohol y otras drogas suele pasar desapercibido en los servicios psiquiátricos, lo cual puede conducir a sobremedicar a los pacientes y formular planes de tratamiento inapropiados, en los que se descuidan intervenciones como desintoxicación, educación y aconsejamiento sobre sustancias de abuso.

Hay pocas evidencias de que los pacientes psiquiátricos puedan mantener un consumo moderado de alcohol u otras drogas, durante largos períodos de tiempo, sin volver a presentar problemas relacionados con el consumo excesivo. Por tanto, deben ser evaluados cualquier consumo de sustancias y cualquier antecedente personal de problemas relacionados con el alcohol o las drogas, ya que con frecuencia les resulta más fácil hablar de sus pautas de consumo en el pasado que de su consumo actual. Para su cribaje, se pueden utilizar cuestionarios de detección de consumo excesivo o de síntomas de dependencia del alcohol.

De acuerdo con los criterios DSM, si el consumo de alcohol es persistente e induce problemas sociales, vocacionales, psicológicos o físicos, debería ser considerado como abuso o dependencia. En los pacientes psiquiátricos, pequeñas cantidades de alcohol o drogas pueden inducir problemas psicológicos o reaparición de los síntomas de enfermedad mental, que pueden evolucionar hacia una clara drogodependencia (32).

Las personas sin hogar presentan diagnóstico dual en un $10 \%-20 \%$, debido a su elevado riesgo de perder el apoyo familiar y el hogar estable. Además, suelen presentar abuso de otras drogas, enfermedades médicas, problemas legales, antecedentes de alteraciones conductuales, traumatismos, deficientes habilidades sociales y vocacionales, así como una pobre red de apoyo. Suelen sufrir malestar psicológico y desmoralización, detenciones por la policía, encarcelamientos, aislamiento de sus familiares y victimización.
Su retención en tratamiento es particularmente difícil. Suelen presentar conductas desadaptadas, como intimidar o amenazar a otras personas, que les son necesarias para sobrevivivir en la calles, pero que van a dificultar su participación en los programas de recuperación, que simpre son a largo plazo, incluso durante años.

\subsection{Trastornos psicóticos inducidos por alcohol}

Determinados trastornos psicóticos pueden ser inducidos por el alcohol:

-Delirium

-Alucinosis

- Trastorno delirante (paranoia, celotipia)

- Intoxicación alcohólica idiosincrática

Además, el abuso de alcohol suele asociarse al de otras drogas (psico-estimulantes, alucinógenos, fenciclidina, a otros trastornos médicos (SIDA, hemorragia cerebral, coma hepático, etc.), y puede asociarse a la epilepsia del lóbulo temporal, que también puede inducir trastornos psicóticos orgánicos. El diagnóstico se efectúa a través de la información de familiares o amigos, exploración física y neurológica, síntomas de intoxicación o abstinencia (característicos de cada sustancia), analítica general (de urgencia), determinación de sustancias en la orina (de urgencia) y neuroimagen (TAC, RM, SPECT).

En la elaboración del diagnóstico dual conviene tener en cuenta la edad de inicio de la dependencia del alcohol, edad de inicio del trastorno psiquiátrico, edad de la primera abstinencia prolongada (más de 3 meses) y la evolución del trastorno psiquiátrico, durante los períodos de abstinencia prolongada (17).

En los pacientes politoxicómanos, el diagnóstico dual debe tener en cuenta la sustancia o sustancias que contribuyeron al inicio del trastorno psiquiátrico, las que producen una reactivación de los síntomas psiquiátricos y la evolución del trastorno psiquiátrico durante los períodos de abstinencia prolongada (confirmada por detecciones periódicas de etanol y otras sustancias en orina). 
El delirium tremens es un trastorno mental orgánico que cursa con un síndrome confusional, trastornos perceptivos (ilusiones o alucinaciones), interpretaciones delirantes y alteraciones emocionales secundarias, agitación psicomotriz e insomnio, síntomas vegetativos y alteraciones hidro-electrolíticas.

La alucinosis alcohólica cursa con alucinaciones auditivas y/o visuales (de contenido amenazador, acusatorio, insultante), ideas delirantes persecutorias (secundarias, pero sistematizadas), ansiedad intensa y riesgo de auto o hetero-agresión (en "defensa propia").

El trastorno delirante cursa con ideas delirantes, de tipo persecutorio o celotípico, riesgo de auto o hetero-agresión, que puede ser contra la pareja, cuando las ideas delirantes son de celos. La intoxicación alcohólica idiosincrática es un grave trastorno de conducta, de tipo agresivo o violento, que se produce tras una pequeña ingesta de alcohol, la cual no produciría intoxicación en la mayoría de personas.

La hemorragia cerebral, la hipoglicemia y la encefalopatía hepática, pueden cursar con un estado confusional que conviene diferenciar del delirium tremens, la encefalopatía de Wernicke $u$ otras encefalopatías alcohólicas y que cuando se acompañan de agitación psicomotriz pueden confundirse con otros trastornos psicóticos.

La intoxicación alcohólica idiosincrásica, que cursa con un estado de agitación psicomotriz, tras el consumo de una pequeña cantidad de alcohol, puede estar acompañada de una reducción del campo de la conciencia, que puede tener un cierto parecido con los estados de agitación debidos a la epilepsia del lóbulo temporal, con la cual ha sido relacionada por algunos autores.

\subsection{Deterioro cognitivo inducido por al- cohol}

En la clasificación DSM IV se consideran la demencia persistente, el trastorno amnéstico persistente inducidos por alcohol y los otros trastornos cognoscitivos.

En la etiología de los trastornos cognoscitivos, asociados al alcoholismo, intervienen diversos factores causales, como son la desnutrición e hipovitaminosis, la neurotoxicidad del etanol y el acetaldehído, las alteraciones metabólicas de la intoxicación o la abstinencia y las alteraciones en la neurotransmisión por aminoácidos excitadores.

Los déficits cognitivos más frecuentes son la alteración de las funciones viso-perceptivas, la pérdida de capacidad para el razonamiento abstracto y las dificultades en la resolución de problemas, capacidad de aprendizaje y de memorización.

Suelen ser déficits transitorios y reversibles, que experimentan una importante mejoría a las 3-6 semanas después de dejar de beber. Con la abstinencia prolongada el paciente puede seguir mejorando, aunque más lentamente.

Los tests neuropsicológicos son el mejor instrumento diagnóstico, para evaluar tanto la severidad del deterioro, como su evolución hacia la mejoría, cuando el paciente se mantiene abstinente, consiguiendo una mayor precisión que las técnicas de neuroimagen.

El trastorno amnéstico inducido por alcohol puede ser transitorio, por intoxicación aguda, en forma de laguna amnéstica o también persistente, es el llamado síndrome de Korsafoff, que suele aparecer tras la encefalopatía aguda de Wernicke.

La demencia alcohólica es un trastorno orgánico persistente que cursa con deterioro de la memoria y una o más alteraciones cognoscitivas, del tipo afasia, apraxia, agnosia, alteración de la ejecución (planificación, organización, secuenciación y abstracción) y deterioro significativo de la actividad laboral o social.

El deterioro en la comprensión, aprendizaje a partir de la experiencia y memorización de experiencias pasadas, junto con otros déficits en el control de impulsos, planificación de la conducta, expresión inadecuada de las emociones, etc., van a condicionar las posibilida- 
des de recuperación del paciente, ya que aumentan el riesgo de recaída.

\section{DIAGNÓSTICO DE LOS TRATORNOS PSIQUIÁTRICOS ASOCIADOS AL ALCO- HOLISMO}

Schuckit (11) aconseja efectuar un análisis cronológico retrospectivo desde la infancia y precisar la edad de (1) inicio del abuso o dependencia del alcohol (2) períodos de abstinencia de varios meses (o años) (3) inicio de trastornos psiquiátricos mayores (no de síntomas psiquiátricos aislados).

Si los síndromes psiquiátricos no precedieron el inicio de la dependencia o abuso de alcohol o no persistieron durante más de 4 semanas de abstención continuada, nos indica que el alcoholismo es el problema mayor y que los síndromes psiquiátricos asociados, aunque pueden ser importantes, son transitorios y se pueden considerar "inducidos" por la intoxicación o por la abstinencia del alcohol.

El alcoholismo primario es un trastorno mental que tiene una evolución espontánea predictible que se conoce como historia natural del alcoholismo, de manera que determinadas señales suelen aparecer a una edad determinada:

13-15 años: inicio del consumo de alcohol

15-17 años: primera intoxicación alcohólica aguda

16-22 años: primer problema relacionado con el alcohol

25-40 años: inicio de la dependencia del alcohol, marcado por graves dificultades relacionadas con el consumo excesivo de alcohol, como:

-ruptura de una relación significativa

-detención por conducir embriagado

-evidencia de abstinencia del alcohol -advertencia por un médico que el alcohol perjudica su salud

-interferencia significativa con el funcionamiento laboral o académico.

Esta progresión cronológica nos puede ayudar en el diagnóstico diferencial del alcoholismo primario, siempre que tengamos en cuenta que los episodios de consumo excesivo se pueden alternar con otros episodios transitorios de abstención continuada o de consumo moderado, de pocas semanas o meses de duración, pero que a la larga van a conducir a la recaída en el consumo excesivo.

El A.S.I. ("Addiction Severity Index") es un instrumento diagnóstico que nos permite evaluar la severidad de la dependencia, obteniendo un perfil de su funcionamiento en diversas áreas: estado médico general, situación laboral, consumo de drogas, consumo de alcohol, situación legal, relaciones personales, adaptación familiar y estado psicológico (34). Su repetición, unos meses después, permite comprobar los progresos en las diversas áreas, además de los cambios en el consumo de las diversas sustancias.

Se trata de una medición objetiva de los progresos del paciente, que nos permite obtener conclusiones, cuando parece que no mejora, y comprobar si ya ha empezado a progresar en su recuperación. Una pregunta que, por otro lado, nos hacen siempre sus familiares y para responder a la cual necesitamos una evaluación polidimensional, que no tenga únicamente en cuenta el consumo de sustancias o las alteraciones conductuales del paciente, sino también otros aspectos de su adaptación, estado médico y psicológico, bienestar personal, etc. Además se van a tener en cuenta las incidencias en su evolución, como pueden ser su asistencia a las actividades terapéuticas programadas, el grado de cumplimiento de la medicación y los acontecimientos no deseados, como separación de la pareja, detención por la policía, etc. 


\subsection{Diagnóstico diferencial}

Cuando el paciente presenta antecedentes de traumatismo cráneo-encefálico o pérdida de conocimiento post-traumática, conviene hacer además una exploración electroencefalográfica, alguna exploración de neuroimagen (estructural o funcional) y una evaluación neuropsicológica o del posible deterioro cognitivo. Algunos trastornos de personalidad pueden ser de etiología orgánica.

El trastorno orgánico de personalidad puede ser secundario a una etiología traumática, infecciosa, vascular, etc. Cursa con alteraciones emocionales (labilidad emocional, euforia superficial e injustificada, cambios rápidos de humor hacia la irritabilidad, ira y agresividad; o bien síntomas de apatía y abulia. Una toma de decisiones impulsiva, sin tener en consideración las posibles consecuencias, que le lleva a cometer actos antisociales. Suspicacia, ideación paranoide o preocupación excesiva por un tema abstracto. Alteraciones del lenguaje del tipo circunstancialidad, sobre-inclusividad, pegajosidad, hipergrafía, etc. Disminución de la sexualidad o cambio del objeto de preferencia sexual. Incluye el síndrome del lóbulo frontal, trastorno de personalidad de la epilepsia límbica y personalidad orgánica pseudopsicopática (35).

El trastorno de personalidad antisocial (TPAS), la esquizofrenia y el trastorno bipolar I suelen aparecer antes del inicio del alcoholismo y suelen ser verdaderos trastornos comórbidos.

La presencia de un trastorno psiquiátrico mayor independiente, como TPAS, esquizofrenia o trastorno bipolar I, va a imponer el curso característico de dichos trastornos psiquiátricos y va a empeorar el pronóstico del alcoholismo, al igual que también lo hace el abuso de otras sustancias psicotrópicas.

\section{TRATAMIENTO DE LA PATOLOGÍA PSI- QUIÁTRICA ASOCIADA AL ALCOHOLISMO}

Los objetivos del tratamiento de los trastornos duales son básicamente conseguir la reti- rada del alcohol y las demás sustancias, junto con la estabilización de los demás trastornos médicos y psiquiátricos.

Se pueden producir situaciones de riesgo elevado que requieren tratamiento inmediato y conviene discriminar si la urgencia es prioritariamente médica, psiquiátrica o de ambas patologías a la vez. Las situaciones de riesgo elevado psiquiátrico son aquellas en que la persona puede poner en peligro su propia vida o la de los demás, debido a su conducta violenta e impulsiva, que puede convertirse en extremadamente peligrosa cuando el paciente se encuentra intoxicado.

El objetivo inicial es el de estabilizar la crisis aguda, lo cual puede requerir la hospitalización psiquiátrica involuntaria. Por otro lado, las emergencias sociales como quedarse sin hogar, victimización o no poder atender las necesidades básicas, requieren una intervención social urgente. Las necesidades biopsico-sociales deberían ser abordadas de manera global.

El programa de tratamiento debería estar fundamentado en una buena relación terapéutica. La intervención psicoterapéutica inicial se orienta hacia persuadir y motivar al paciente para que se implique y comprometa con el programa. El terapeuta tendrá que explorar y averiguar lo que el paciente desea y necesita.

Resulta imprescindible un programa integrado en el que un único equipo especializado, tanto en drogodependencias como en salud mental, atienda simultáneamente toda la patología mental del paciente, tanto desde el punto de vista farmacoterapéutico, como psicoterapéutico, como su integración familiar y socio-ocupacional (36).

Tanto los abordajes breves e intensivos, como los abordajes disociados en dos redes de atención especializada, están condenados al fracaso terapéutico. El paciente dual requiere un proceso terapéutico que va a durar varios meses, antes de que consiga estabilizarse y puede ser perjudicado por la disparidad entre los enfoques diagnósticos y terapéuticos de los profesionales que le 
atienden en un centro de salud mental y los que le atienden en el centro de drogodependencias.

Un único equipo de profesionales bien entrenados, tanto en conductas adictivas como en salud mental, cuya intervención sea integrada, no de tipo secuencial ni tampoco en paralelo; que sea sensible a las particularidades culturales, étnicas, de nacionalidad, religión y espiritualidad de cada paciente, son las cualidades que pueden ofrecer un mayor rendimiento terapéutico.

Además de un intensa formación, el equipo requiere supervisión continuada, como algo habitual, no solamente en los momentos de crisis del equipo, sino precisamente con la finalidad de prevenir dichas crisis. Por otro lado, dicho equipo tendrá que desarrollar su propia filosofía, teniendo en cuenta unos valores y objetivos que mejoren su cohesión interna.

La recuperación es un proceso lento, que cursa con etapas de remisión, que se alternan con múltiples episodios de crisis o recaídas. El paciente requiere reevaluación periódica y cambios en el plan terapéutico, a medida que se van descifrando, con su ayuda, las claves de su compleja problemática. El proceso de recuperación se podría comparar con el de atravesar un laberinto. El profesional ya lo ha recorrido con otros pacientes pero cada vez es un poco diferente. Sabemos a dónde queremos llegar pero no por dónde tendremos que pasar esta vez. Son las decisiones que el paciente va a tomar, de manera consecutiva, las que nos van a llevar por nuevos y desconocidos itinerarios. Sólo la intuición del profesional, que ya ha conseguido encontrar la salida al laberinto, en anteriores ocasiones, permitirá superar las situaciones decisivas. De lo contrario podemos entrar en una situación de atasco o desorientación, como si fueramos dando vueltas en círculo, en un sector limitado, repitiendo de manera estereotipada decisiones erróneas, que suelen llevar al paciente a la recaída o a la reagudización de su psicopatología.

Resulta necesario disponer de una unidad de intervención en crisis, que atienda al paciente y sus familiares afectados, a cualquier hora del día o de la noche y que pueda ofrecer hospitalización inmediata al paciente, incluso en contra de su voluntad y con autorización judicial, cuando el paciente presente una descompensación psicótica, suicida o se encuentre gravemente intoxicado y corra el riesgo de accidente, victimización o abuso de las personas más cercanas.

El paciente con patología dual requiere un abordaje conductual y psicoeducativo, pero no psicodinámico. El abordaje psicoterapéutico confrontativo va a aumentar la defensividad del paciente y los intentos de facilitar su introspección van a producirle un intenso malestar emocional que puede disparar un estado de "craving" de beber o tomar otras drogas o medicación tranquilizante.

Los objetivos de la prevención de recaídas son la detección de los signos de recaída, la identificación de las causas de la recaída y el desarrollo de estrategias de intervención específica, para interrumpir el proceso de recaída. Las experiencias estresantes habituales, que no suelen alterar el funcionamiento de una persona saludable, pueden tener un intenso impacto en el paciente con diagnóstico dual y pueden llevarle a la recaída.

Las determinaciones de sustancias en la orina, el test de alcohol en el aire espirado y los marcadores biológicos de consumo excesivo de alcohol permiten monitorizar el posible consumo de sustancias del paciente. La determinación de niveles plasmáticos de fármacos permite conocer su cumplimiento terapéutico y si los niveles del fármaco son los más apropiados.

El aprendizaje de técnicas de relajación, meditación, biofeedbak, prevención de recaídas y ejercicio físico moderado, permiten una reducción progresiva de la medicación.

El paciente y sus familiares necesitan información e intercambiar experiencias con otros pacientes y familiares, acerca del tratamiento. La necesidad de tomar medicación, sus efectos terapéuticos esperables, los posibles efectos adversos, el tiempo aproximado que tendrá que tomar cada fármaco, las decisio- 
nes de instaurar o retirar el medicamento, los fármacos con mayor o menor potencial adictivo, los efectos del consumo de alcohol o drogas, asociado a determinados medicamentos (riesgo de sobredosis, accidentes, lesiones, etc.).

El consentimiento informado conviene utilizarlo antes de la prescripción de un fármaco potencialmente peligroso, como el disulfiram, y garantiza que tanto el paciente como sus familiares han comprendido con detalle las características del fármaco. La dosificación ideal, para obtener un buen cumplimiento, es la de una vez al día, o mejor todavía, los medicamentos de acción prolongada.

La desintoxicación de sustancias, en régimen de hospitalización, parcial o completa, permite una más exhaustiva exploración diagnóstica, una mejor supervisión y cumplimiento de la medicación, que puede ser monitorizada además con mayor precisión, y una intervención más intensiva, para conseguir la toma de conciencia y la motivación del paciente, conseguir su compromiso y retención en el programa terapéutico y educarle sobre la relación entre el consumo de alcohol y los síntomas psiquiátricos.

Dado que el riesgo de suicidio y el de hepatotoxicidad puede ser especialmente elevado en los pacientes alcohólicos con patología dual, conviene supervisar y monitorizar intensivamente la medicación que toman.

El tratamiento ambulatorio convencional suele ser insuficiente para el tratamiento de la patología dual. Se requiere la hospitalización completa inicial para efectuar una completa evaluación diagnóstica, el tratamiento de desintoxicación de alcohol y otras drogas y el inicio de tratamiento y estabilización de la patología psiquiátrica. Posteriormente a la hospitalización completa conviene que el paciente pase por una hospitalización parcial o por un medio residencial, tipo comunidad terapéutica, para conseguir la estabilización completa de la psicopatología y la reducción o abandono estables del consumo de alcohol y otras drogas. Posteriormente se podría pasar a un seguimiento intensivo individual, grupal y familiar, junto con determinaciones frecuentes de drogas en orina, cobertura psicofarmacológica (no adictiva), técnicas cognitivo-conductuales, grupos de prevención de recaídas y grupos con los familiares.

Se trata de un abordaje a largo plazo y por etapas, que debe durar años, mas que semanas o meses y conviene utilizar intervenciones motivacionales previamente a las intervenciones orientadas hacia la abstinencia. Se han propuesto cuatro etapas que tienen una cierta superposición:

1. Compromiso: desarrollar relaciones de confianza o una alianza de trabajo.

2. Persuasión: ayudar al paciente a percibir y reconocer las consecuencias adversas del consumo de sustancias y desarrollar su motivación hacia la recuperación.

3. Tratamiento activo: ayudarle a conseguir la remisión estable, bien sea mediante consumo controlado o bien mediante abstención continuada, que sería la mejor opción.

4. Prevención de recaídas: ayudarle a mantenerse en remisión estable.

Es preferible trabajar con el paciente en su ambiente natural y si acaso recurrir al ingreso breve, en unidades de desintoxicación, lo cual va a facilitar el diagnóstico, el desarrollo de una relación terapéutica y el inicio de la tarea de motivación del paciente; para pasar después a la hospitalización parcial, que contribuya a consolidar tanto el compromiso del paciente como su persuasión y toma de conciencia del problema.

El número de pacientes en remisión estable va aumentando con el paso de los años de tratamiento y aproximadamente el $50 \%$ consiguen la abstención continuada cuando llevan unos tres años de tratamiento (32).

\subsection{Farmacoterapia del alcoholismo aso- ciado a patología psiquiátrica}

Algunos medicamentos tienen un cierto efecto reforzador, que no tendría mayor importancia en una persona sana pero que puede resultar decisivo para que un paciente 
drogodependiente haga un mal uso, abuso o desarrolle tolerancia, dependencia, y abstinencia o sobredosis de dicho fármaco.

Es comprensible que esto suceda con los fármacos psicoestimulantes (anfetaminas, metilfenidato) y con los fármacos opiáceos (meperidina, morfina, codeína), pero para muchos profesionales resulta desconocido e inesperado que un paciente con un trastorno dual desarrolle abuso o dependencia de fármaco hipno-sedativos o antiparkinsonianos.

Las benzodiazepinas son fármacos psicoactivos, que inducen refuerzo conductual, tolerancia, abstinencia y tienen potencial de abuso. Sobretodo entre los pacientes drogodependientes, que pueden tener una respuesta diferente al resto de la población, tanto al alcohol como a las benzodiazepinas (36).

Cuando se trata de un paciente depresivo psicótico o esquizofrénico descompensado, se requiere la administración rápida y a dosis elevadas de psicofármacos, en régimen de hospitalización, para evitar auto o heteroagresiones; siempre acompañado del tratamiento de desintoxicación apropiado, en función de las diversas sustancias que toma el paciente. Sin embargo, una vez superada la intervención en crisis o cuando se presenten otros trastornos psiquiátricos, de menor severidad, es preferible recurrir a fármacos que no sean psicoactivos, ni reforzadores de la conducta de auto-administración, como buspirona, beta-bloqueantes, antidopaminérgicos y eutimizantes.

Una vez superada la primera etapa de desintoxicación, es preferible no prescribir benzodiazepinas (BZD) e intentar retirarlas con una pauta muy lentamente decreciente, cuando el paciente se ha convertido ya en abusador o dependiente. Las benzodiazepinas de vida media corta, como alprazolam o lorazepam, pueden ser sustituídas por otras de vida media más prolongada, como clonazepam, clorazepato o bromazepam, y retiradas progresivamente.

Tanto Imipramina como Desipramina han demostrado su eficacia para el tratamiento de de pacientes alcohólicos que presentan depresión, pero los antidepresivos tricíclicos presentan un peligroso potencial de sobredosis letal en los alcohólicos deprimidos, por el riesgo de inducir arritmias cardíacas, que se puede evitar utilizando inhibidores selectivos de recaptación de monoaminas (serotonina y noradrenalina). Los inhibidores de la monoamino-oxidasa (IMAO) están contraindicados en los pacientes alcohólicos

Los inhibidores selectivos de recaptación de serotonina han demostrado ser eficaces para reducir tanto los síntomas depresivos como la conducta de beber en exceso en los alcohólicos deprimidos.

El trastorno bipolar parece tener una mejor respuesta a los eutimizantes del grupo de los anticonvulsivantes, como valproato o carbamazepina y una menor respuesta al Litio, que además tiene una mayor potencial tóxico en sobredosis.

Buspirona ha demostrado mejorar la retención en tratamiento y disminuir la frecuencia de consumo de alcohol, en pacientes con elevados niveles de ansiedad. Además, carece de potencial adictivo y no tiene efectos depresores sinérgicos, cuando se asocia al consumo de alcohol. Sus inconvenientes pueden ser la latencia de la respuesta terapéutica ansiolítica, entorno a las 2 semanas, la necesidad de dosis más elevadas en los pacientes alcohólicos (entorno a los 60 $\mathrm{mg} / \mathrm{día}$ ) y la tendencia a un bajo cumplimiento de la pauta prescrita (8). El entrenamiento en relajación, la práctica de ejercicio físico moderado y los cuidados higiénico-dietéticos pueden contribuir a mejorar tanto la ansiedad, como el sueño, como el estado de ánimo, de manera significativa, en el paciente alcohólico en recuperación.

Los beta-bloqueantes, como propanolol, pueden atenuar las descargas adrenérgicas de las crisis de angustia, bloqueando el componente somático de la ansiedad. También pueden disminuir el temblor y la inquietud. No son fármacos psicoactivos, no tienen efectos reforzadores y no alteran el ánimo. Dado que desarrollan tolerancia, pierden eficacia con su administración continuada, por lo que se recomienda utilizarlos para prevenir 
determinadas situaciones que el paciente anticipe que pueden ser angustiosas, durante un episodio de ansiedad o en la etapa final de la retirada de BZD, para combatir síntomas como temblor, taquicardia, angustia, etc. Descartando siempre previamente sus posibles contraindicaciones, como las crisis asmáticas o bradicardia.

Los antihistamínicos, como difenhidramina, doxilamina, hidroxicina, etc., pueden ser utilizados como hipno-inductores, aprovechando sus efectos sedativos, pero el rápido desarrollo de tolerancia reduce su eficacia a largo plazo, por lo que es preferible reservarlos para cuando se retiran la BZD hipno-inductoras, durante un tiempo limitado y pasar después a su utilización opcional, no diaria.

Los antipsicóticos atípicos, como olanzapina o quetiapina y los antidopaminérgicos sedativos, que inducen pocos efectos extrapiramidales (tiapride, tioridazina, etc.) pueden ser utilizados también como inductores del sueño y pueden mejorar la disforia, paranoidismo, hostilidad interpersonal, control de impulsos, inestabilidad emocional, ansiedad y somatizaciones. Además podrían tener un cierto efecto anti-craving y reducir el riesgo de conductas impulsivas que pueden conducir a la recaída.

Los eutimizantes del tipo anticomiciales (valproato, carbamazepina, topiramato, gabapentina, etc.) pueden estabilizar el estado de ánimo del paciente y permiten prescindir incluso de la utilización de otros fármacos como antidepresivos, en pacientes cuyos estados de estrés, conflico interpersonal, malestar emocional o craving, induzcan alteraciones transitorias del estado de ánimo. Además, podrían atenuar los síntomas de "flashback" relacionados con el consumo de drogas o con el trastorno de estrés postraumático y tampoco tienen riesgo de abuso o dependencia. Los pacientes alcohólicos presentan una mayor incidencia de traumatismos cráneo-encefálicos que pueden dejar como secuela alteraciones comiciales del tipo epilepsia del lóbulo temporal, difícimente detectables con el electroencefalograma convencional. Cuando las posibles alteraciones emocionales y conductuales del paciente tienen un trasfondo de alteración bioeléctrica, debido a una antigua lesión cerebral, se puede obtener una buena respuesta terapéutica con anticomiciales. Dado que el Litio y la Carbamazepina requieren una monitorización frecuente y conllevan el riesgo de graves efectos adversos, es preferible utilizar los demás anticomiciales.

El Disulfiram, a dosis de 250 mg/día, cuya eficacia requiere de una administración supervisada, puede ser una gran ayuda cuando el paciente presenta comorbilidad psiquiátrica o adictiva, se propone no tomar bebidas alcohólicas y siempre que el fármaco consiga reforzar dicho propósito. Por otro lado, no parece aumentar significativamente el riesgo de empeoramiento de la sintomatología del paciente psicótico ni el de interacciones con los psicofármacos (37). Además, algunos estudios han evaluado su posible utilidad terapéutica cuando el paciente presenta una dependencia asociada de cocaína o cuando se encuentra en programa de mantenimiento con metadona.

Naltrexona y acamprosato son fármacos que pueden reducir el consumo de alcohol, la pérdida de control tras un consumo inicial $y$, en ocasiones, incluso el deseo de beber (38).

\subsection{Otras estrategias terapéuticas}

La monitorización de los niveles plasmáticos de los psicofármacos administrados puede ser una buena medida para comprobar el cumplimiento terapéutico, por parte del paciente, verificar si los niveles plasmáticos son terapéuticos a las dosis habituales, o bien si va a requirir dosis mayores que las habituales para obtener niveles plasmáticos realmente terapéuticos; o también vigilar que niveles plasmáticos excesivamente elevados pudieran ocasionar efectos adversos.

Las determinaciones periódicas de etanol, benzodiazepinas y otras drogas en la orina, así como la determinación de etanol en el aire espirado (alcotest) nos permiten conocer la intensidad y frecuencia del consumo de 
sustancias por parte del paciente, durante su recuperación.

La psicoterapia cognitivo-conductual ha demostrado su eficacia para el tratamiento, tanto del alcoholismo, como para la depresión, fobias, angustia, trastorno obsesivocompulsivo, etc. La terapia conductual dialéctica podría ser eficaz para el tratamiento del trastorno límite de personalidad. En general, la sinergia entre dichas psicoterapias especializadas y la fármacoterapia consiguen mejores resultados que cada una de ellas por separado.

La psicoterapia individual y grupal, orientadas hacia la reducción del consumo de sustancias y, si es posible, su total abstinencia, estará orientada hacia la prevención de recaídas y la intervención en crisis, cuando se produzca dicha recaída, con la finalidad de evitar que arrastre al paciente a un episodio de auto-destrucción o al abandono del programa terapéutico. La colaboración con los familiares puede resultar decisiva para prevenir recaídas o cuando menos, detectarlas precozmente y minimizar sus consecuencias destructivas. Los grupos con familiares pueden ser de utilidad para mejorar la comunicación con el paciente, cambiar dinámicas familiares patológicas y diseñar un plan de tratamiento de cara al alta del paciente hospitalizado (39).

\section{CONCLUSIONES}

Aproximadamente el $50 \%$ de pacientes con enfermedades mentales graves, tales como esquizofrenia o trastorno bipolar, desarrollan un trastorno por abuso de sustancias, a lo largo de su vida. Esta tasa es probablemente más elevada entre los jóvenes con antecedentes de violencia o que duermen en la calle y entre los pacientes atendidos en servicios de urgencias.

Suelen presentar además una pobre adaptación en diversas áreas de su funcionamiento, resultados de tratamiento desfavorables e incidencias como quedarse sin hogar, hospitalizaciones o ingresos en prisión.

Con frecuencia, el consumo perjudicial o el abuso de sustancias, pasa desapercibido a los cínicos que trabajan en salud mental. Los procedimientos estandarizados de cribaje y evaluación diagnóstica dual, podrían mejorar este problema.

Para optimizar el rendimiento terapéutico convendría hacer una planificación apropiada del tratamiento, tanto del abuso de sustancias como de la comorbilidad psiquiátrica. La intervención terapéutica debería ser efectuada por profesionales expertos en patología dual y en el marco de una unidad especializada en el abordaje de dichas patologías, que pueda integrar el tratamiento del trastorno mental grave, con el del abuso de sustancias y siempre a largo plazo.

\section{REFERENCIAS BIBLIOGRAFICAS}

(1) WOODY G: The challenge of dual diagnosis. Alcohol Health \& Research World 1996; 20: 7680.

(2) REGIER DA; FARMER MA; RAE DS; LOCKE BZ; KEITH SJ; JUDD LL; GOODWIN FK: Comorbidity of mental disorder with alcohol mand other drug abuse. Results from the epidemiologic catchment area (ECA) study. JAMA 1990; 264:2511-2518.

(3) KESSLER RC; McGONAGLE KA; ZHAO S; NELSON CB; HIGES m; ESHLEMAN S; WITTCHENHU; KENDLER KS: Lifetime and 12month prevalence of DSM-III-R psychiatric disorders in the United States. Arch. Gen. Psychiatry 1994; 51:8-19.

(4) HELZER JE; PRYZBECK TR: The co-occurence of alcoholism with other psychiatric disorders in the general population and its impact on treatment. J. Stud. Alcohol, 1988; 49: 219-224.

(5) KUSHNER MG; SHER KJ; ERICKSON DJ: Prospective analysis of the relation between DSMIII anxiety disorders and alcohol use disorders.

Am. J. Psychiatry 1999; 156:723-732.

(6) DRIESSEN M,; VELTRUP C; WETTERLING T; JOHN U; DILLING H: Axis I and Axis II comorbidity in alcohol dependence and the two types 
of alcoholism. Alcohol. Clin. Exp. Res. 1998; 22: 77-86.

(7) MERIKANGAS KR; STEVENS D; FENTON B: Comorbidity of alcoholism and anxiety disorders. Role of family studies. Alcohol Health \& Research World 1996; 20: 100-106.

(8) MODESTO-LOWE V; KRANZLER HR: Diagnosis and treatment of alcohol-dependent patients with comorbid psychiatric disorders. Alcohol Health \& Research World 1999; 23:144-149.

(9) ASOCIACIÓN AMERICANA DE PSIQUIATRÍA: DSM IV. Manual diagnóstico y estadístico de los trastornos mentales. Barcelona. Masson. 1995.

(10) KENDLER KS; WALTERS EE; NEALE MC; KESSLER RC, HEALTH AC; EAVES LJ: The structure of the genetic and environmental risk factors for six major psychiatric disorders in women. Phobia, generalized ansiety disorder, panic disorder, bulimia, major depression, and alcoholism. Arch. Gen. Psyciatry 1995; 52: 374-383.

(11) SCHUCKIT MA: Alcohol, anxiety, and depressive disorders. Alcohol Health \& Research World 1996; 20: 81-86.

(12) BERGLUND M; ÖJEHAGEN A: The influence of alcohol drinking and alcohol use disorders on psychiatric disorders and suicidal behavior. Alcohol. Clin. Exp. Res. 1998; 22: 333S-345S.

(13) ANTON RF: What is craving? Models and implications for treatment. Alcohol Research \& Health 1999; 23: 165-173.

(14) SCHUCKIT MA; IRWIN M; SMITH TL: Oneyear incidence rate of major depression and other psychiatric disorders in 139 alcoholic men. Addiction 1994; 89: 441-445.

(15) MERIKANGAS KR; LECKMAN JF; PURSOFF BA; PAULS DL; WEISSMAN MM: Familial transmission of depression and alcoholism. Arch. Gen. Psychiatry 1985; 42:367-372.

(16) BROWN SA; SCHUCKIT MA: Changes in depression among abstinent male alcoholics.

J. Stud. Alcohol. 1988; 52:55-61.

(17) SCHUCKIT MA; TIPP JE; BUCHOLZ KK; NURNBERGERE Jl; HESSELBROCK VM; CROWE RR; KRAMER J: The life-time rates of three major mood disorders and four major anxiety disorders in alcoholics and controls. Addiction 1997; 92:1289-1304.

(18) ROY A; DE JONG J; LAMPARSKI D; GEORGE D; LINNOILA M: Mental disorders among alco- holics. Arch. Gen. Psychiatry 1991; 48: 428432.

(19) ROY A; LAMPARSKI D; DE JONG J; et al.: Characteristics of alcoholics who attempt suicide. American Journal of Psychiatry 1990; 147: 781-785.

(20) HASSIN DS; TSAI W-Y; ENDICOTT J; MUELLER TI; CORYELL W; KELLER M: The effects of major depression on alcoholism. Five-year course. Am. J. Addict. 1996; 5: 144-155.

(21) BROWN SA; INABA RK; GILLIN JC; SCHUCKIT MA; STEWART MA; IRWIN MR: Alcoholism and affective disorder: clinical course of depresive symptoms. Am. J. Psychiatry. 1995; 152: 45-52.

(22) MONRAS M; MARCOS V; RAMON G: Tentativas de suicidio en pacientes alcohólicos. Adicciones 1992; 4: 45-56.

(23) HIMLE JA; HILL EM: Alcohol abuse and the anxiety disorders: evidence from Epidemiologic Catchment Area survey. J. Anxiety Disorders 1991; 5: 237-245.

(24) ROSS HE; GLASER FB; GERMANSON T: The prevalence of psychiatric disorders in patients with alcohol and other drug problems. Arch. Gen. Psychiatry 1998; 45: 1023-1031.

(25) STOCKWELL T: Alcohol withdrawal: an adaptation to heavy drinking of no practical significance? Addiction 1994; 89: 1447-1453.

(26) HESSELBROCK MN, MEYER R; KEENER JJ: Psychopathology in hospitalized alcoholics. Arch. Gen. Psychiatry 1985; 42: 1050-1055.

(27) MILLER WR; BENNETT ME: Treating alcohol problems in the context of other drug abuse. Alcohol Health \& Research World 1996; 20:118-123.

(28) SLUTSKE WS; EISEN S; TRUE WR; LYONS MJ; GOLDBERG J; TSUANG M: Common genetic vulnerability for pathological gambling and alcohol dependence in men. Arch. Gen. Psychiatry 2000; 57: 666.673.

(29) KESSLER RC; CRUM RM; WARNER LA; NELSON CB; SCHULENBERG J; ANTHONY JC: Lifetime co-occurence of DSM-III-R alcohol abuse and dependence with other psychiatric disorders in the National Comorbidity Survey. Arch. Gen. Psychiatry 1997; 54:313-321.

(30) LILENFELD LR; KAYE W: The link between alcoholism and eating disorders. Alcohol Health \& Research World. 1996; 20:94-99. 
(31) HIGUCHI S; SUZUKI K; YAMADA K; PARRISH $\mathrm{K}$; KONO H: Alcoholics with eating disorders: prevalence and clinical course-a study from Japan. British Journal of Psychiatry. 1993; 162: 403-406.

(32) DRAKE RE; MUESER KT: Alcohol-use disorder and severe mental illness. Alcohol Health \& Research World. 1996; 20:87-93.

(33) CUFFEL BJ: Comorbid substance use disorder. Prevalence, patterns of use, and course. In: Drake RE and Mueser KT eds. Dual diagnosis of major mental illness and substance disorder: recent research and clinical implications. San Francisco: Jossey-Bass, 1996. Pp. 93-105.

(34) McLELLAN AT; PARIKH G; BRAGG A, CACCIOLA J; FUREMAN B; INCMIKOFI R: AddictionSeverity Index Administration Manual. $5^{\text {th }}$ ed. Philadelphia. Veterans Affairs Center for Studies of Addiction, 1990.

(35) ORGANIZACIÓN MUNDIAL DE LA SALUD (O.M.S.): Trastornos Mentales y del Comportamiento. Décima revisión de la Clasifica- ción Internacional de Enfermedades CIE 10. Madrid. MEDITOR. 1992.

(36) SUBSTANCE ABUSE AND MENTAL HEALTH SERVICES ADMINISTRATION (SAMHSA) CENTER FOR SUBSTANCE ABUSE TREATMENT: Assessment and treatment of patients with coexisting mental ilness and alcohol and other drug abuse. Tratment Improvement Protocol (TIP) Series 9. U. S. Rockville, MD. Department of Health and Human Services. 1994.

(37) LARSON EW; OLINCY A, RUMMANS TA, MORSE RM: Disulfiram in the treatment of patients with both alcohol dependence and other psychiatric disorders: a review. Alcohol. Clin. Exp. Res. 1992; 16: 125-130.

(38) GUARDIA SERECIGNI, J.: Naltrexona en el tratamiento del alcoholismo. Psiquiatría Biológica. $1998 ; 5$ : 35-44.

(39) DACKIS ChA; GOLD MS: Psychiatric hospitals for treatment of dual diagnosis. In: Lowinson JH, Ruiz P, Millman R, Langrod JG (Eds.) Substance Abuse. A comprehesive textbook. Baltimore. Williams \& Wilkins. 1992 (p. 467-485). 